\title{
On the Pricing of Exotic Options: a New Closed-Form Valuation Approach
}

\author{
Stelios Bekiros* \\ European University Institute, Florence, Italy \\ Dimitra Kouloumpou ${ }^{\dagger}$ \\ Athens University of Economics and Business, Athens, Greece
}

February 12, 2019

\begin{abstract}
We provide a novel method to estimate in a closed-form solution the option prices of various exotic options, using techniques based on Laplace-Beltrami operator for estimating diffusion boundary times. We estimate exit times and their expectations, the hitting probabilities, boundary local times until the first hitting and other probabilistic quantities and moment generating functions related to local hitting times. Our findings maybe of paramount importance for traders, investors, speculators and more broadly speaking for financial institutions.
\end{abstract}

Mathematics Subject Classification (2000): 60-08; 60J75; 47A68; 42A85; 91B28

Mathematics Subject Classification (2010): 91G20; 91B25; 60J28; 47H25; 46F12

Keywords: Brownian motion; option pricing; crossing probabilities; diffusion approximations JEL codes: G10; G12; G13; C63

\section{Introduction}

The payoff of a simple European or American-style call or put option while it depends heavily upon the value of the underlying asset, yet not particularly so on the path taken. In general, options derivatives products are determined by many features and primarily upon the underlying assets, as commonly reported in the relevant literature. However, a plethora of exotic options including Binary options, Cashor-Nothing, Asset-or-Nothing, Barrier, Double Barrier options amongst other, all depend strongly on the path of the asset as well and on whether price barriers are hit or not. These barriers eventually control the option valuation. Once either of these barriers is breached, the status of the option is immediately determined, namely either the option comes into existence in case the barrier - as called - is in- or knockin barrier, or ceases to exist if the barrier is out- or knock-out barrier. Other double barrier options of many types also exist (see [1] ). In this work, we utilize double barrier options as proper proxies for many categories of exotic derivatives. To the best of our knowledge, we present for the first time new ways of estimating the expectation of the time when various exotic options seize to exist, their hitting probabilities, the exit times and their expectations, boundary local times until the first hitting and other probabilistic quantities related to the boundary local times. Importantly, we deliver closed-form solutions

The diversity of exotic barrier options is indicative of their applicability in modern derivatives markets. Many different combinations of barrier options can be implemented in derivatives markets for equities, FX, commodities and bonds. We present thereafter the most important types: a double knock-out (DKO)

\footnotetext{
${ }^{*}$ European University Institute, Department of Economics, Via della Piazzuola; 43, I-50133, Florence, Italy; E-mail address: stelios.bekiros@eui.eu;

${ }^{\dagger}$ Athens University of Economics and Business. Email: dkouloumpou@aueb.gr.
} 
or one touch knock-out Barrier option has both lower and upper knock-out barriers. Initially the holder of the option owns a call or a put. If at any time, either barrier is breached, the option seizes to exist (knocked-out). In some cases at knock-out, the holder may receive a rebate. For a double knock-in (DKI), or one touch knock-in barrier, if either barrier is breached, the holder of the barrier option is knocked-in, hence now owns the call or put. In cases where the option is never knocked-in, the holder may receive a rebate. An upper barrier knock-out (UKO) double barrier involves the upper barrier which when breached prior to the lower barrier, the option holder is knocked out, whilst in case the lower is breached prior to the upper or neither barrier is breached, the holder owns the option. Furthermore, in case of an upper barrier knock-out double option (UKO2) if the upper barrier is breached prior to the lower barrier, the option holder gets nothing and instead in case the lower barrier is breached prior to the upper, the holder receives an option. All in all, if neither barrier is breached, the holder gets nothing. For a lower barrier knock-out (LKO) if the lower barrier is hit prior to the upper barrier, the holder is knocked out and when the upper barrier is hit prior to the lower or neither barrier is breached, the holder owns an option. Additionally, for double lower barrier knock-outs (LKO2) if the lower barrier is breached prior to the upper one, the option holder gets nothing while when the upper barrier is breached prior to the lower, the holder receives the option. If neither barrier is breached, the holder is not compensated. An upper barrier knock-in double barrier (UKI) relates to the case whereby, if the upper barrier is reached prior to the lower barrier, the option holder receives a call or put, whilst in case neither barrier is hit, the holder owns an option. A lower barrier knock-in (LKI) performs similarly to the UKI, yet with a switch on the lower and upper barrier. A double touch knock-out option (DTKO) exists when the holder initially holds a call or put option. However, if both the upper and lower barriers are breached during the life of the option, the holder is knocked out. For a double touch knock-in option (DTKI), if both the upper and lower barriers are breached during the life of the option, the holder is knocked-in to a call or put option. With all of the aforementioned barrier variations, the specification of rebate is possible. These rebates (cash or asset amounts) can be specified if one or the other barrier is hit or if neither barrier is reached. Using these rebate features is a way of including digital / binary payoffs that depend on barrier levels. Finally, the type of monitoring conducted upon the barriers is a very important feature as well. Several possibilities exist, namely each barrier is continuously monitored for the life of the option or the barrier is partially monitored for specific windows during the option life. During these windows, the barriers are monitored continuously. Alternatively, each barrier is partially monitored for specific windows during the life of the option and the barriers are monitored at discrete dates or in another case the barrier is discretely monitored at specific dates.

Merton ([2]) was the first to derive a closed form solution for a down-and-out European call option. Other closed-form pricing formulae of exotic derivatives i.e., particularly for single-barrier options were published by Rubinstein and Reiner ( [3] ). Rich ([4]) provided a mathematical framework for pricing the single-barrier options. A valuation method for double-barrier options based upon the probabilistic approach was discussed by Kunitomo and Ikeda ([5]). The values of the double barrier options can also be obtained by solving the Black-Scholes partial differential equation with the corresponding boundary conditions using the method of separation of variables. Analytical solutions of one-touch double-barrier binary options, in which a fixed payoff is determined by whether it is touching the barrier, are derived by Hui ([6]). Hui ([7]) extends regular single and double barrier options to time-dependent barrier options in which the barrier period covers a segment of time either at the beginning (front end) or the end (rear end) of the option life. This feature makes the time-dependent barrier options more flexible than the regular barrier options for an investor, having a particular view on an underlying asset in a certain period of time. The one-time barrier discontinuity in the time-dependent barrier options makes their pricing formulae different from the regular barrier option-pricing formulae. Roberts and Shortland ([8]) consider the problem of pricing derivative securities which involve a barrier clause. They present general techniques to calculate, or estimate accurately barrier option prices, using methods for estimating diffusion process with hitting times. Mario Dell'Era ([9]) discusses the efficiency of the spectral method for computing the 
value of double barrier options. Using this method, one may write the option price as a Fourier series with suitable coefficients. However, all of the aforementioned methods cannot be generalized vis-a-vis the valuation of many other exotic derivatives, and more importantly they do not tackle with all the analytical closed-form specificities occuring and remain unsolvable.

We contribute to the literature in significant ways. Specifically, to our knowledge this is the first study to estimate a closed-form solution for all the inherent features of a variety of barrier options and other proxies. Specifically, i) the expectation of the time that the option dies out is calculated, ii) the hitting probabilities i.e., the probabilities that the option hits first the upper or the lower barrier are accurately estimated, iii) certain probabilistic quantities related to the boundary local time until first hitting are introduced, iv) the exit times and their expectations are estimated as well as v) the boundary local times until the first hitting, which are of immense importance to the investors, alongside with the moment generating functions for all of the above. In this work, we consider as our proxy the double knock-out barrier with two barriers related to the strike price: an upper and a lower one. The upper barrier defines the level where the trigger price is above the strike price, while the lower barrier establishes a point at which the trigger price is below the strike. If the underlying does not break out of either barrier at any time during the option life, the option acts like a plain vanilla option and the holder would receive a specified payout. However, if one of the barriers has been broken through, the option dies out (gets knocked-out). Our novel concept is that we assume that the underlying asset follows a geometric Brownian motion on a 1 -dimensional sphere $S^{1}=\left\{x=(a \cos \varphi, a \sin \varphi) \in \mathbb{R}^{2} \mid 0 \leq \varphi<2 \pi\right\}$ (i.e. circle) with center at the origin and radius $a>\frac{H_{2}}{2 \pi}$. In this case, the transformation introduces $\varphi_{0}, \varphi_{1} \in[0,2 \pi)$ such that $H_{1}=a \varphi_{0}$ and $H_{2}=a \varphi_{1}$ i.e., two points on the circle, that denote the upper and lower barriers. The underlying asset then starts from a point $\phi \in D=\left(H_{1}, H_{2}\right)$. Via this method, we estimate the closed-form solution of the price of every barrier option or any exotic one thereby. Moreover, we calculate the expectation of the time the option dies out. The probabilities that the option hits first the upper or the lower barrier are calculated and we evaluate certain probabilistic quantities related to the boundary local time of the domain $D$ until first hitting. We deliver valuable closed-form mathematical solutions of paramount importance for traders, investors, speculators and more broadly speaking for financial institutions. The paper is organized as follows: section 2 presents preliminary definitions, propositions and proofs, whilst section 3 describes the valuation method and a theorem related to that. Section 4 recalls some definitions and proofs, and presents new results on exit times, expectations, hitting probabilities and moments generating fnctions. Section 5 exposes the proofs for estimating boundary local times. Finally, section 6 concludes with very interesting remarks regarding the extension of the results on spheres of higher dimensions and future applications of the presented methodology to the valuation of other exotic derivatives as well as to other mathematical problems in many topical fields.

\section{Preliminaries}

\subsection{The $n$-Sphere $S^{n}$}

Definition 2.1. Let $n \in \mathbb{N}^{*}=\{1,2,3, \ldots\}$. The $n$-dimensional sphere $S^{n}$ with center $\left(c_{1}, c_{2, \ldots}, c_{n+1}\right)$ and radius $a>0$ is (defined to be) the set of all points $x=\left(x_{1}, x_{2}, \ldots, x_{n+1}\right) \in \mathbb{R}^{n+1}$ satisfying $\left(x_{1}-c_{1}\right)^{2}+$ $\left(x_{2}-c_{2}\right)^{2}+\ldots+\left(x_{n+1}-c_{n+1}\right)^{2}=a^{2}$. Thus,

$$
S^{n}=\left\{\left(x_{1}, x_{2}, \ldots, x_{n+1}\right) \in \mathbb{R}^{n+1} \mid\left(x_{1}-c_{1}\right)^{2}+\left(x_{2}-c_{2}\right)^{2}+\ldots+\left(x_{n+1}-c_{n+1}\right)^{2}=a^{2}\right\}
$$

The points of the $n$-sphere with center at the origin and radius $a$ for $n=1$ may also be discribed in spherical coordinates in the following way

$$
S^{1}=\left\{x=(a \cos \varphi, a \sin \varphi) \in \mathbb{R}^{2} \mid 0<\varphi \leq 2 \pi\right\}
$$


The Laplace-Beltrami operator of a smooth function $f$ on $S^{1}$ is

$$
\Delta_{1} f=\frac{1}{a^{2}} \frac{\partial^{2} f}{\partial \varphi^{2}}
$$

(see $[10])$.

\subsection{Brownian Motion on $S^{n}$}

Definition 2.2. The Brownian motion on $S^{n}$ is a diffusion (Markov) process $X_{t}, t \geq 0$, on $S^{n}$ whose transition density is a function $P(t, x, y)$ on $(0,+\infty) \times S^{n} \times S^{n}$ satisfying

$$
\begin{gathered}
\frac{\partial P}{\partial t}=\frac{1}{2} \Delta_{n} P \\
P(t, x, y) \rightarrow \delta_{x}(y) \text { as } t \rightarrow 0^{+}
\end{gathered}
$$

where $\Delta_{n}$ is the Laplace-Beltrami operator on $S^{n}$ acting on the $x$-variables and $\delta_{x}(y)$ is the delta mass at $x$, i.e. $P(t, x, y)$ is the heat kernel of $S^{n}$. The heat kernel exists, it is unique, positive and smooth in $(t, x, y)$.

\subsubsection{Further Properties of the Heat Kernel $P(t, x, y)$}

Moreover the heat kernel possesses the following properties:

1. Symmetry in $x, y$, that is $P(t, x, y)=P(t, y, x)$.

2. The semigroup identity: For any $s \in(0, t)$

$$
P(t, x, y)=\int_{S^{n}} P(s, x, z) P(t-s, z, y) d \mu z
$$

where $d \mu$ is the area measure element of $S^{n}$.

3. As $t \rightarrow \infty, P(t, x, y)$ approaches the uniform density on $S^{n}$, i.e. $\lim _{t \rightarrow \infty} P(t, x, y)=\frac{1}{A_{n}}$ where $A_{n}$ is the area of the $S^{n}$ with radius $a$. It is well known that

$A_{n}=\frac{2 \pi \frac{n+1}{2} a^{n}}{\left(\frac{n-1}{2}\right) !}$ for $n$ odd and $A_{n}=\frac{2^{n}\left(\frac{n}{2}-1\right) ! \pi^{\frac{n}{2}} a^{n}}{(n-1) !}$ for $n$ even

4. Finally, the symmetry of $S^{n}$ implies that $P(t, x, y)$ depends only on $t$ and $d(x, y)$, the distance between $x$ and $y$. In spherical coordinates it depends on $t$ and the angle $\varphi$ between $\mathrm{x}$ and $\mathrm{y}$. Hence $P(t, x, y)=P(t, \varphi)$ where $P(t, \varphi)$ satisfies

$$
\frac{\partial P}{\partial t}=\frac{1}{2} \Delta_{n} P=\frac{1}{2 a^{2}}\left[(n-1) \cot \varphi \frac{\partial P}{\partial \varphi}+\frac{\partial^{2} P}{\partial \varphi^{2}}\right]
$$

and

$$
\lim _{t \rightarrow 0^{+}} a A_{n-1} P(t, \varphi) \sin ^{n-1} \varphi=\delta(\varphi)
$$

The symbol $\delta(\cdot)$ denotes the standard Dirac delta function on $\mathbb{R}$. 


\subsubsection{Explicit Form of the Heat Kernel of $S^{1}$}

Reminder (Poisson Summation Formula). Let $f(x)$ be a function in the Schwartz space $\mathcal{S}(\mathbb{R})$, where $\mathcal{S}(\mathbb{R})$ consists of the set of all infinitely differentiable functions $f$ on $\mathbb{R}$ so that $f$ and all its derivatives $f^{(l)}$ are rapidly decreasing, in the sense that

$$
\sup _{x \in \mathbb{R}}|x|^{k}\left|f^{(l)}(x)\right|<\infty \quad \text { for every } \quad k, l \geq 0 .
$$

Then

$$
\sum_{n \in \mathbb{Z}} f(x+2 \pi n)=\frac{1}{2 \pi} \sum_{n \in \mathbb{Z}} F(n) \exp (i n x),
$$

where $F(\xi)$ is the Fourier transform of $f(x)$, i.e.

$$
F(\xi)=\int_{-\infty}^{+\infty} f(x) \exp (-i \xi x) d x, \quad \xi \in \mathbb{R} .
$$

For example, if

$$
f(x)=\exp \left(-A x^{2}+B x\right), \quad A>0, \quad B \in \mathbb{C},
$$

then

$$
F(\xi)=\sqrt{\frac{\pi}{A}} \exp \left(\frac{(i \xi-B)^{2}}{4 A}\right)
$$

\subsection{The Case of $S^{1}$}

Proposition 2.1. The transition density function of the Brownian motion $X_{t}, t \geq 0$ on $S^{1}$ with radius $a$ is the function

$$
p(t, \varphi)=\frac{1}{2 \pi a} \sum_{n \in \mathbb{Z}} \exp \left(-\frac{n^{2} t}{2 a^{2}}+i n \varphi\right) .
$$

Equivalently

$$
p(t, \varphi)=\frac{1}{\pi a} \sum_{n \in \mathbb{N}}\left[\exp \left(-\frac{n^{2} t}{2 a^{2}}\right) \cos (n \varphi)\right]-\frac{1}{2 \pi a} .
$$

and

$$
p(t, \varphi)=\frac{1}{\sqrt{2 \pi t}} \sum_{n \in \mathbb{Z}} \exp \left(-\frac{a^{2}}{2 t}(\varphi-2 \pi n)^{2}\right) .
$$

Proof. If

$$
p(t, \varphi)=\frac{1}{\pi a} \sum_{n \in \mathbb{N}}\left[\exp \left(-\frac{n^{2} t}{2 a^{2}}\right) \cos (n \varphi)\right]-\frac{1}{2 \pi a},
$$

then

$$
\frac{\partial p(t, \varphi)}{\partial t}=-\frac{1}{2 \pi a^{3}} \sum_{n \in \mathbb{N}} n^{2} \cos (n \varphi) \exp \left(-\frac{n^{2} t}{2 a^{2}}\right)
$$

and

$$
\frac{\partial^{2} p(t, \varphi)}{\partial \varphi^{2}}=-\frac{1}{\pi a} \sum_{n \in \mathbb{N}} n^{2} \cos (n \varphi) \exp \left(-\frac{n^{2} t}{2 a^{2}}\right) .
$$

Therefore

$$
\frac{\partial p(t, \varphi)}{\partial t}=\frac{1}{2 a^{2}} \frac{\partial^{2} p(t, \varphi)}{\partial \varphi^{2}} .
$$

We will now show that

$$
\lim _{t \rightarrow 0^{+}} a p(t, \varphi)=\delta(\varphi) .
$$

If $\varphi \in(0,2 \pi)$, then

$$
\lim _{t \rightarrow 0^{+}} a p(t, \varphi)=0 .
$$


Next we observe that

$$
\int_{0}^{2 \pi} a p(t, \varphi) d \varphi=\frac{1}{\pi} \int_{0}^{2 \pi} \sum_{n \in \mathbb{N}}\left[\exp \left(-\frac{n^{2} t}{2 a^{2}}\right) \cos (n \varphi)\right] d \varphi-1 .
$$

For $t>0$ let us consider the functions

$$
f_{n}:[0,2 \pi] \rightarrow \mathbb{R}, \quad n \in \mathbb{N}
$$

with

$$
f_{n}(\varphi)=\cos (n \varphi) \exp \left(-\frac{n^{2} t}{2 a^{2}}\right) .
$$

Notice that $f_{n}(\varphi)$ are integrable functions on $[0,2 \pi]$. Furthermore

$$
\sum_{n=1}^{+\infty} f_{n}(\varphi)
$$

converges uniformly on $[0,2 \pi]$ because

$$
\left|f_{n}(\varphi)\right| \leq \exp \left(-\frac{n^{2} t}{2 a^{2}}\right)
$$

and the series

$$
\sum_{n=1}^{\infty} \exp \left(-\frac{n^{2} t}{2 a^{2}}\right)
$$

converges. Therefore (2.15) gives

$$
\int_{0}^{2 \pi} a p(t, \varphi) d \varphi=-1+\frac{1}{\pi} \sum_{n \in \mathbb{N}} \exp \left(-\frac{n^{2} t}{2 a^{2}}\right) \int_{0}^{2 \pi} \cos (n \varphi) d \varphi
$$

thus

$$
\int_{0}^{2 \pi} a p(t, \varphi) d \varphi=1, \text { for every } t>0 .
$$

Therefore from (2.15) and (2.16)

$$
\lim _{t \rightarrow 0^{+}} a p(t, \varphi)=\delta(\varphi)
$$

and this complete the proof.

\subsection{Geometric Brownian Motion on a 1-dimentional Sphere $S^{1}$}

Definition 2.3. Let $X_{t}, t \geq 0$ be the Brownian motion on $S^{1}$ of radius a. The geometric Brownian motion on $S^{1}$ of radius a with drift is

$$
Z_{t}=Z_{0} \exp \left[\left(r-\frac{1}{2} \sigma^{2}\right) t+\sigma a X_{t}\right]
$$

i.e. $Z_{t}$ have stochastic differential $d Z_{t}=r Z_{t} d t+\sigma a Z_{t} d X_{t}$.

We have already shown that, the Brownian motion on $S^{1}$ of radius $a$, in spherical coordinates is the solution of the stochastic differntial equation $d X_{t}=\frac{1}{a} d B_{t}$. Hence $d Z_{t}=r Z_{t} d t+\sigma Z_{t} d B_{t}$. Therefore, the generator $L$ of $Z_{t}$ is given by

$$
L f(\varphi)=r \varphi \frac{\partial f}{\partial \varphi}+\frac{1}{2} \sigma^{2} \varphi^{2} \frac{\partial^{2} f}{\partial \varphi^{2}}
$$




\subsection{Transition Density Function of the Geometric Brownian Motion on $S^{1}$}

Let $X_{t}, t \geq 0$ be the Brownian Motion on $S^{1}$ of radius $a$.The transition density function of the Brownian motion $X_{t}, t \geq 0$ on $S^{1}$ of radius $a$ is the function (2.9) i.e.

$$
p(t, \varphi)=\frac{1}{2 \pi a} \sum_{n \in \mathbb{Z}} \exp \left(-\frac{n^{2} t}{2 a^{2}}+i n \varphi\right)
$$

This means that $F_{X_{t}}(\varphi)=P\left[X_{t} \leq \varphi\right]=\int_{0}^{\varphi} p(t, \varphi) d \varphi$. The geometric Brownian Motion $Z_{t}, t \geq 0$ on $S^{1}$ of radius $a$ is $Z_{t}=Z_{0} \exp \left[\left(r-\frac{1}{2} \sigma^{2}\right) t+\sigma a X_{t}\right]$.Hence,

$F_{Z_{t}}(\varphi)=P\left[Z_{t} \leq \varphi\right]=P\left[Z_{0} \exp \left[\left(r-\frac{1}{2} \sigma^{2}\right) t+\sigma a X_{t}\right] \leq \varphi\right]=P\left[X_{t} \leq \frac{1}{\sigma a} \ln \left(\frac{\varphi}{Z_{0}}\right)+\left(\frac{\sigma}{2 a}-\frac{r}{\sigma a}\right) t\right]=$ $\int_{-\infty}^{\frac{1}{\sigma a} \ln \left(\frac{\varphi}{Z_{0}}\right)+\left(\frac{\sigma}{2 a}-\frac{r}{\sigma a}\right) t} p(t, y) d y$.

Now differentiating with respect to $\varphi$, we obtain that the transition density function of the geometric Brownian Motion, is the function

$$
p_{z_{t}}(t, \varphi)=\frac{1}{\sigma a \varphi} p\left(t, \frac{1}{\sigma a} \ln \left(\frac{\varphi}{Z_{0}}\right)+\left(\frac{\sigma}{2 a}-\frac{r}{\sigma a}\right) t\right)
$$

i.e.

$$
p_{z_{t}}(t, \varphi)=\frac{1}{2 \pi a^{2} \sigma \varphi} \sum_{n \in \mathbb{Z}} \exp \left(-\frac{n^{2} t}{2 a^{2}}+i n\left(\frac{1}{\sigma a} \ln \left(\frac{\varphi}{z_{0}}\right)+\frac{\sigma^{2}-2 r}{2 \sigma a} t\right)\right)
$$

\section{$3 \quad$ Value of the derivative security}

We limit ourselves to assume that the underlying asset follows a Geometric Brownian motion with drift, i.e. $d Y_{t}=r Y_{t} d t+\sigma Y_{t} d B_{t}$, where $Y_{t}$ is the asset price and $B_{t}, t \geq 0$ is the Brownian motion.

Define $\Psi\left(Y_{T}\right)=\left(Y_{T}-k\right)^{+}=\max \left\{\left(Y_{T}-k\right), 0\right\}$ be the payoff of the derivative security at time $T$ if the underlying security is at $Y_{T}$ ( $k$ is the strike price of the option). Assume that there is a double knock-out Barrier at levels $H_{1}, H_{2} \in \mathbb{R}$ such that $H_{1}<H_{2}$. i.e., if one of the barrier is reached in a double knock-out option, the option is killed. The idea is to consider the geometric Brownian motion on a 1 -dimensional sphere $S^{1}=\left\{x=(a \cos \varphi, a \sin \varphi) \in \mathbb{R}^{2} \mid 0 \leq \varphi<2 \pi\right\}$ (circle) with center at the origin and radius $a>\frac{H_{2}}{2 \pi}$.

In this case there exist $\varphi_{0}, \varphi_{1} \in[0,2 \pi)$ such that $H_{1}=a \varphi_{0}$ and $H_{2}=a \varphi_{1}$ and $k=a \varphi_{k}$. For arbitrary process $S$ and $H_{1}, H_{2} \in \mathbb{R}$ such that $H_{1}<H_{2}$, we use the following notation according to ([8]):

$$
\begin{gathered}
\tau_{H_{1}}^{Y}=\inf \left\{t: Y(t) \leq H_{1}\right\} \text { if } Y(0)>H_{1} \text { and } \\
\tau_{H_{2}}^{Y}=\inf \left\{t: Y(t) \geq H_{2}\right\} \text { if } Y(0)<H_{2}
\end{gathered}
$$

where $H_{1}$ and $H_{2}$ are the Barriers.

Let $Y(t)$ be the value of the stock at time $t \in[0, T]$, where $T=\min \left\{\tau_{H_{1}}^{y}, \tau_{H_{2}}^{y}\right\}$.From the theory of arbitrage-free pricing in a complete market (see [12]), the value of the derivative security can then be expressed as follows

$$
V(t, x)=V\left(T, H_{1}, H_{2}, Y(t), t\right)=E\left[\Psi\left(S_{T}\right) I\left(\tau_{H_{1}}^{y}<T\right) I\left(\tau_{H_{2}}^{y}<T\right)\right.
$$

where $\Psi\left(Z_{T}\right)=\left(Y_{T}-k\right)^{+}=\max \left\{\left(Y_{T}-k\right), 0\right\}$ is the payoff of the derivative security at time $T$ if the underlying security is at $Y_{T}$. The boundary problem for $V(t, x)$ can be tackled with fast and accurate pricing of Barrier options under Levy processes to solve it 


$$
\left\{\begin{array}{c}
\frac{\partial V}{\partial t}+L V-r V=0 \\
V(0, x)=\left(x_{0}-k\right)^{+} \\
V\left(t, H_{1}\right)=V\left(t, H_{2}\right)=0 \\
V(t, x)=0 \text { for every } x \in\left(-\infty, H_{1}\right] \cup\left[H_{2},+\infty\right)
\end{array}\right\}
$$

where $L$ is the generator of $Y_{t}$.

In case $Z_{t}$ is the Geometric Brownian motion without drift on a 1-dimensional sphere $S^{1}$ of radius $a>\frac{H_{2}}{2 \pi}$, i.e. $d Z_{t}=\sigma a Z_{t} d X_{t}$ and we let $\phi_{1}, \varphi_{2} \in[0,2 \pi)$, such that $\varphi_{1}<\varphi_{2}$ with $a \varphi_{1}=H_{1}$ and $\varphi_{2}=H_{2}$, then the problem (3.2) is equivelant to

$$
\left\{\begin{array}{c}
\frac{\partial u}{\partial t}+r \varphi \frac{\partial u}{\partial \varphi}+\frac{1}{2} \sigma^{2} \varphi^{2} \frac{\partial^{2} u}{\partial \varphi^{2}}-r u=0 \\
u(0, \varphi)=a\left(\varphi_{0}-\varphi_{k}\right)^{+} \\
u\left(t, \varphi_{1}\right)=u\left(t, \varphi_{2}\right)=0 \\
u(t, \varphi)=0 \text { for every } \varphi \in D^{c}, \text { where } D=\left(\varphi_{1}, \varphi_{2}\right) \subset S^{1}
\end{array}\right\}
$$

where $a \varphi_{0}=x_{0}$ and $a \varphi_{k}=k$

Theorem 3.1. Under Black-Scholes framework the arbitrage-price of a knock-out call double barrier option is given by relation

$$
\begin{aligned}
V(t, x)= & \int_{0}^{\ln \left(\frac{H_{2}}{H_{1}}\right)} \frac{2 \exp [-r(T-t)]}{\ln \left(\frac{H_{2}}{H_{1}}\right)}\left(e^{\xi} H_{1}-k\right)^{+} I_{[A(t)<\xi<B(t): t \in[0, T]]} . \\
& \sum_{n \in \mathbb{Z}}\left[\exp \left[\frac{(n \pi \sigma)^{2}(T-t)}{2 \ln ^{2}\left(\frac{H_{2}}{H_{1}}\right)}\right] \sin \left(\frac{n \pi \xi}{\ln \left(\frac{H_{2}}{H_{1}}\right)}\right) \sin \left(\frac{n \pi \ln \left(\frac{x}{H_{1}}\right)}{\ln \left(\frac{H_{2}}{H_{1}}\right)}\right)\right] d \xi
\end{aligned}
$$

where $A(t)=\ln H_{1}+(T-t)\left(r-\frac{\sigma^{2}}{2}\right)$ and $B(t)=\ln H_{2}+(T-t)\left(r-\frac{\sigma^{2}}{2}\right)$ (see [8])

\section{Exit Times}

We recall some basic definitions:

Definition 4.1. A measurable space $\{\Omega, F\}$ is said to be equipped with a filtration $\left\{F_{t}\right\}, t \in[0,+\infty)$, if for every $t \geq 0\left\{F_{t}\right\}$ is a $\sigma$-algebra of subsets of $\Omega$ such that $F_{t} \subset F$ and for every $t_{1}, t_{2} \in[0,+\infty)$ such that $t_{1}<t_{2}$, we have that $F_{t_{1}} \subset F_{t_{2}}$. (i.e. $\left\{F_{t}\right\}$ is an increasing family of sub $\sigma$-algebras of $F$ ).

Definition 4.2. Let us consider a measurable space $\{\Omega, F\}$ equipped with a filtration $\left\{F_{t}\right\}$. A random variable $T$ is a stopping time with respect to the filtration $\left\{F_{t}\right\}$, if for every $t \geq 0 \quad\{\omega \in \Omega \mid T(\omega) \leq t\} \in$ $F_{t}$.

Let $Z_{t}$ be the Geometric Brownian motion on $S^{1}$ and $D \subset S^{1}$ a domain. Then $T=\inf \left\{t \geq 0 \mid Z_{t} \notin D\right\}$ is a stopping time with respect to $F_{t}=\sigma\left\{Z_{s} \mid 0 \leq s \leq t\right\}$, called the exit time on $\partial D$.(For more details see [13]).

\subsection{Expectations of Exit Times on $S^{1}$}

Proposition 4.1. Let $\varphi_{1}, \varphi_{2} \in(0,2 \pi]$, such that $\varphi_{1}<\varphi_{2}$, both fixed. We consider the set $D$ in $S^{1}$, such that $D=\left(\varphi_{1}, \varphi_{2}\right)$. If $Z_{t}$ is the Geometric Brownian motion with drift on $S^{1}$ of radius a starting at the 
point $\varphi \in D$, then the expectation of $T$ is given by

$$
E^{\varphi}[T]=\frac{2}{\sigma^{2}-2 r} \cdot \frac{\left(\varphi_{2}^{\frac{\sigma^{2}-2 r}{\sigma^{2}}}-\varphi_{1}^{\frac{\sigma^{2}-2 r}{\sigma^{2}}}\right) \ln \varphi-\left(\varphi_{2}^{\frac{\sigma^{2}-2 r}{\sigma^{2}}}-\varphi^{\frac{\sigma^{2}-2 r}{\sigma^{2}}}\right) \ln \varphi_{1}-\left(\varphi^{\frac{\sigma^{2}-2 r}{\sigma^{2}}}-\varphi_{1}^{\frac{\sigma^{2}-2 r}{\sigma^{2}}}\right) \ln \varphi_{2}}{\left(\varphi_{2}^{\frac{\sigma^{2}-2 r}{\sigma^{2}}}-\varphi_{1}^{\frac{\sigma^{2}-2 r}{\sigma^{2}}}\right)}
$$

Proof. Reminder. If $u(x)=E^{x}[T]$, then $u(x)$ satisfies

$$
\begin{gathered}
L u(\varphi)=-1 \\
\left.u\right|_{\partial D}=0
\end{gathered}
$$

(see [14])

Hence from (2.18) the differential equation takes the form

$$
r \varphi \frac{\partial u}{\partial \varphi}+\frac{\sigma^{2} \varphi^{2}}{2} \frac{\partial^{2} u}{\partial \varphi^{2}}=-1
$$

with boundary condition

$$
u\left(\varphi_{1}\right)=u\left(\varphi_{2}\right)=0
$$

From (4.2) and (4.3) we imply that

$u(\varphi)=\frac{2}{\sigma^{2}-2 r} \cdot \frac{\left(\varphi_{2}^{\frac{\sigma^{2}-2 r}{\sigma^{2}}}-\varphi_{1}^{\frac{\sigma^{2}-2 r}{\sigma^{2}}}\right) \ln \varphi-\left(\varphi_{2}^{\frac{\sigma^{2}-2 r}{\sigma^{2}}}-\varphi^{\frac{\sigma^{2}-2 r}{\sigma^{2}}}\right) \ln \varphi_{1}-\left(\varphi^{\frac{\sigma^{2}-2 r}{\sigma^{2}}}-\varphi_{1}^{\frac{\sigma^{2}-2 r}{\sigma^{2}}}\right) \ln \varphi_{2}}{\left(\varphi_{2}^{\frac{\sigma^{2}-2 r}{\sigma^{2}}}-\varphi_{1}^{\frac{\sigma^{2}-2 r}{\sigma^{2}}}\right)}$

i.e.

$E^{\varphi}[T]=\frac{2}{\sigma^{2}-2 r} \cdot \frac{\left(\varphi_{2}^{\frac{\sigma^{2}-2 r}{\sigma^{2}}}-\varphi_{1}^{\frac{\sigma^{2}-2 r}{\sigma^{2}}}\right) \ln \varphi-\left(\varphi_{2}^{\frac{\sigma^{2}-2 r}{\sigma^{2}}}-\varphi^{\frac{\sigma^{2}-2 r}{\sigma^{2}}}\right) \ln \varphi_{1}-\left(\varphi^{\frac{\sigma^{2}-2 r}{\sigma^{2}}}-\varphi_{1}^{\frac{\sigma^{2}-2 r}{\sigma^{2}}}\right) \ln \varphi_{2}}{\left(\varphi_{2}^{\frac{\sigma^{2}-2 r}{\sigma^{2}}}-\varphi_{1}^{\frac{\sigma^{2}-2 r}{\sigma^{2}}}\right)}$

Based on the proof above, if $Y_{t}$ is the asset price and we have a double knock-out barrier at level $H_{1}=a \varphi_{1}$ and $H_{2}=a \varphi_{2}$ then if its price starts at the point $x \in\left(H_{1}, H_{2}\right)$ the expectation of $T=$ $\inf \{t \geq 0 \mid$ the option is killed $\}$ is

$$
E^{x}[T]=\frac{2}{\sigma^{2}-2 r} \cdot \frac{\left(H_{2}^{\frac{\sigma^{2}-2 r}{\sigma^{2}}}-H_{1}^{\frac{\sigma^{2}-2 r}{\sigma^{2}}}\right) \ln x-\left(H_{2}^{\frac{\sigma^{2}-2 r}{\sigma^{2}}}-x^{\frac{\sigma^{2}-2 r}{\sigma^{2}}}\right) \ln H_{1}-\left(x^{\frac{\sigma^{2}-2 r}{\sigma^{2}}}-H_{1}^{\frac{\sigma^{2}-2 r}{\sigma^{2}}}\right) \ln H_{2}}{\left(H_{2}^{\frac{\sigma^{2}-2 r}{\sigma^{2}}}-H_{1}^{\frac{\sigma^{2}-2 r}{\sigma^{2}}}\right)}
$$

\subsection{Expectation of $f\left(Z_{t}\right)$}

Proposition 4.2. Let $\varphi_{1}, \varphi_{2} \in(0,2 \pi]$, such that $\varphi_{1}<\varphi_{2}$, both fixed. We consider the set $D$ in $S^{1}$, such that $D=\left(\varphi_{1}, \varphi_{2}\right)$. If $Z_{t}$ is the Geometric Brownian motion with drift on $S^{1}$ of radius a starting at the point $\varphi \in D$, and $f$ be a function on $\partial D$, then the expectation of $f\left(Z_{t}\right)$ is given by

$$
E^{\varphi}\left[f\left(Z_{t}\right)\right]=\frac{f\left(\varphi_{2}\right)\left(\varphi^{\frac{\sigma^{2}-2 r}{\sigma^{2}}}-\varphi_{1}^{\frac{\sigma^{2}-2 r}{\sigma^{2}}}\right)+f\left(\varphi_{1}\right)\left(\varphi_{2}^{\frac{\sigma^{2}-2 r}{\sigma^{2}}}-\varphi^{\frac{\sigma^{2}-2 r}{\sigma^{2}}}\right)}{\left(\varphi_{2}^{\frac{\sigma^{2}-2 r}{\sigma^{2}}}-\varphi_{1}^{\frac{\sigma^{2}-2 r}{\sigma^{2}}}\right)}
$$


Proof. It is known that the function $u(\varphi)=E^{\varphi}\left[Z_{t}\right]$ satisfies the differential equation

$$
L u(\varphi)=0
$$

with boundary condition

$$
\left.u\right|_{\partial D}=f
$$

(see [13])

Hence from (2.18) the differential equation takes the form

$$
r \varphi \frac{\partial u}{\partial \varphi}+\frac{\sigma^{2} \varphi^{2}}{2} \frac{\partial^{2} u}{\partial \varphi^{2}}=0
$$

with boundary condition

$$
u\left(\varphi_{1}\right)=f\left(\varphi_{1}\right) \text { and } u\left(\varphi_{2}\right)=f\left(\varphi_{2}\right)
$$

From (4.6) and (4.7) we imply that

$$
\begin{gathered}
u(\varphi)=\frac{f\left(\varphi_{2}\right)\left(\varphi^{\frac{\sigma^{2}-2 r}{\sigma^{2}}}-\varphi_{1}^{\frac{\sigma^{2}-2 r}{\sigma^{2}}}\right)+f\left(\varphi_{1}\right)\left(\varphi_{2}^{\frac{\sigma^{2}-2 r}{\sigma^{2}}}-\varphi^{\frac{\sigma^{2}-2 r}{\sigma^{2}}}\right)}{\left(\varphi_{2}^{\frac{\sigma^{2}-2 r}{\sigma^{2}}}-\varphi_{1}^{\frac{\sigma^{2}-2 r}{\sigma^{2}}}\right)} \\
E^{\varphi}\left[f\left(Z_{t}\right)\right]=\frac{f\left(\varphi_{2}\right)\left(\varphi^{\frac{\sigma^{2}-2 r}{\sigma^{2}}}-\varphi_{1}^{\frac{\sigma^{2}-2 r}{\sigma^{2}}}\right)+f\left(\varphi_{1}\right)\left(\varphi_{2}^{\frac{\sigma^{2}-2 r}{\sigma^{2}}}-\varphi^{\frac{\sigma^{2}-2 r}{\sigma^{2}}}\right)}{\left(\varphi_{2}^{\frac{\sigma^{2}-2 r}{\sigma^{2}}}-\varphi_{1}^{\frac{\sigma^{2}-2 r}{\sigma^{2}}}\right)}
\end{gathered}
$$

\subsection{Hitting Probabilities}

Proposition 4.3. Let $\varphi_{1}, \varphi_{2} \in(0,2 \pi]$, such that $\varphi_{1}<\varphi_{2}$, both fixed. We consider the sets $D_{1}, D_{2}$ in $S^{1}$, such that $D_{1}=\left(\varphi_{1}, 2 \pi\right]$ and $D_{2}=\left(0, \varphi_{2}\right)$. Let $Z_{t}$ is the Geometric Brownian motion with drift on $S^{1}$ of radius a starting at the point $\varphi \in D_{1} \cap D_{2}$. If

$$
\begin{aligned}
& T_{1}=\inf \left\{t \geq 0 \mid Z_{t} \notin D_{1}\right\} \\
& T_{2}=\inf \left\{t \geq 0 \mid Z_{t} \notin D_{2}\right\}
\end{aligned}
$$

and

$$
T=\inf \left\{t \geq 0 \mid Z_{t} \notin D_{1} \cap D_{2}\right\}
$$

then the probabilities $\operatorname{Pr}^{\varphi}\left\{T=T_{1}\right\}$ and $\operatorname{Pr}^{\varphi}\left\{T=T_{2}\right\}$ are given by

$$
\stackrel{\varphi}{\operatorname{Pr}}\left\{T=T_{1}\right\}=\frac{\varphi_{2}^{\frac{\sigma^{2}-2 r}{\sigma^{2}}}-\varphi^{\frac{\sigma^{2}-2 r}{\sigma^{2}}}}{\varphi_{2}^{\frac{\sigma^{2}-2 r}{\sigma^{2}}}-\varphi_{1}^{\frac{\sigma^{2}-2 r}{\sigma^{2}}}}
$$

and

$$
\stackrel{\varphi}{\operatorname{Pr}}\left\{T=T_{2}\right\}=\frac{\varphi^{\frac{\sigma^{2}-2 r}{\sigma^{2}}}-\varphi_{1}^{\frac{\sigma^{2}-2 r}{\sigma^{2}}}}{\varphi_{2}^{\frac{\sigma^{2}-2 r}{\sigma^{2}}}-\varphi_{1}^{\frac{\sigma^{2}-2 r}{\sigma^{2}}}}
$$


Proof. From (4.5) for $f(x)=x$ we have $E^{\varphi}\left[f\left(Z_{t}\right)\right]=\frac{\varphi_{2}\left(\varphi^{\frac{\sigma^{2}-2 r}{\sigma^{2}}}-\varphi_{1}^{\frac{\sigma^{2}-2 r}{\sigma^{2}}}\right)+\varphi_{1}\left(\varphi_{2}^{\frac{\sigma^{2}-2 r}{\sigma^{2}}}-\varphi^{\frac{\sigma^{2}-2 r}{\sigma^{2}}}\right)}{\left(\varphi_{2}^{\frac{\sigma^{2}-2 r}{\sigma^{2}}}-\varphi_{1}^{\frac{\sigma^{2}-2 r}{\sigma^{2}}}\right)}$.

However,

$$
E^{\varphi}\left[f\left(Z_{t}\right)\right]=\varphi_{1} \stackrel{\varphi}{\operatorname{Pr}}\left\{T=T_{1}\right\}+\varphi_{2} \stackrel{\varphi}{\operatorname{Pr}}\left\{T=T_{2}\right\}
$$

and

$$
\stackrel{\varphi}{\operatorname{Pr}}\left\{T=T_{1}\right\}+\stackrel{\varphi}{\operatorname{Pr}}\left\{T=T_{2}\right\}=1
$$

Therefore,

$$
\stackrel{\varphi}{\operatorname{Pr}}\left\{T=T_{1}\right\}=\frac{\varphi_{2}^{\frac{\sigma^{2}-2 r}{\sigma^{2}}}-\varphi^{\frac{\sigma^{2}-2 r}{\sigma^{2}}}}{\varphi_{2}^{\frac{\sigma^{2}-2 r}{\sigma^{2}}}-\varphi_{1}^{\frac{\sigma^{2}-2 r}{\sigma^{2}}}}
$$

and

$$
\stackrel{\varphi}{\operatorname{Pr}}\left\{T=T_{2}\right\}=\frac{\varphi^{\frac{\sigma^{2}-2 r}{\sigma^{2}}}-\varphi_{1}^{\frac{\sigma^{2}-2 r}{\sigma^{2}}}}{\varphi_{2}^{\frac{\sigma^{2}-2 r}{\sigma^{2}}}-\varphi_{1}^{\frac{\sigma^{2}-2 r}{\sigma^{2}}}}
$$

Based on the proof above, in case $Y_{t}$ is the asset price and we have a double knock-out Barrier at levels $H_{1}=a \varphi_{1}$ and $H_{2}=a \varphi_{2}$ then if its price starts at the point $x \in\left(H_{1}, H_{2}\right)$ the probability the option is killed because it reaches the barrier level $H_{1}$ is given as

$$
\stackrel{x}{\operatorname{Pr}}\left\{T=T_{1}\right\}=\frac{H_{2}^{\frac{\sigma^{2}-2 r}{\sigma^{2}}}-x^{\frac{\sigma^{2}-2 r}{\sigma^{2}}}}{H_{2}^{\frac{\sigma^{2}-2 r}{\sigma^{2}}}-H_{1}^{\frac{\sigma^{2}-2 r}{\sigma^{2}}}}
$$

and the proability the option is killed because it reaches the barrier level $H_{2}$ is

$$
\stackrel{x}{\operatorname{Pr}}\left\{T=T_{2}\right\}=\frac{x^{\frac{\sigma^{2}-2 r}{\sigma^{2}}}-H_{1}^{\frac{\sigma^{2}-2 r}{\sigma^{2}}}}{H_{2}^{\frac{\sigma^{2}-2 r}{\sigma^{2}}}-H_{1}^{\frac{\sigma^{2}-2 r}{\sigma^{2}}}}
$$

\subsection{Moment Generating functions}

Proposition 4.4. Let $\varphi_{0}, \varphi_{1} \in(0,2 \pi]$, such that $\varphi_{0}<\varphi_{1}$ both fixed. We consider the set $D$ on $S^{1}$ such that $D=\left(\varphi_{0}, \varphi_{1}\right)$. If $Z_{t}$ is the geometric Brownian motion on $S^{1}$ of radius a starting at the point $\varphi \in D$, then the expectation of $\exp (-\lambda T)$ is given by

$E^{\varphi}[\exp (-\lambda T)]$

$$
\begin{aligned}
& =\frac{\left(\varphi_{2}^{-\frac{\left(2 r-\sigma^{2}\right)+\sqrt{\left(2 r-\sigma^{2}\right)^{2}+8 \lambda \sigma^{2}}}{2 \sigma^{2}}}-\varphi_{1}^{-\frac{\left(2 r-\sigma^{2}\right)+\sqrt{\left(2 r-\sigma^{2}\right)^{2}+8 \lambda \sigma^{2}}}{2 \sigma^{2}}}\right) \varphi^{-\frac{\left(2 r-\sigma^{2}\right)-\sqrt{\left(2 r-\sigma^{2}\right)^{2}+8 \lambda \sigma^{2}}}{2 \sigma^{2}}}}{\varphi_{1}^{-\frac{\left(2 r-\sigma^{2}\right)-\sqrt{\left(2 r-\sigma^{2}\right)^{2}+8 \lambda \sigma^{2}}}{2 \sigma^{2}}} \varphi_{2}^{-\frac{\left(2 r-\sigma^{2}\right)+\sqrt{\left(2 r-\sigma^{2}\right)^{2}+8 \lambda \sigma^{2}}}{2 \sigma^{2}}}-\varphi_{1}^{-\frac{\left(2 r-\sigma^{2}\right)+\sqrt{\left(2 r-\sigma^{2}\right)^{2}+8 \lambda \sigma^{2}}}{2 \sigma^{2}}} \varphi_{2}^{-\frac{\left(2 r-\sigma^{2}\right)-\sqrt{\left(2 r-\sigma^{2}\right)^{2}+8 \lambda \sigma^{2}}}{2 \sigma^{2}}}}+ \\
& +\frac{\left(\varphi_{1}^{-\frac{\left(2 r-\sigma^{2}\right)-\sqrt{\left(2 r-\sigma^{2}\right)^{2}+8 \lambda \sigma^{2}}}{2 \sigma^{2}}}-\varphi_{2}^{\left.-\frac{\left(2 r-\sigma^{2}\right)-\sqrt{\left(2 r-\sigma^{2}\right)^{2}+8 \lambda \sigma^{2}}}{2 \sigma^{2}}\right) \varphi^{-\frac{\left(2 r-\sigma^{2}\right)+\sqrt{\left(2 r-\sigma^{2}\right)^{2}+8 \lambda \sigma^{2}}}{2 \sigma^{2}}}}\right.}{\varphi_{1}^{-\frac{\left(2 r-\sigma^{2}\right)-\sqrt{\left(2 r-\sigma^{2}\right)^{2}+8 \lambda \sigma^{2}}}{2 \sigma^{2}}} \varphi_{2}^{-\frac{\left(2 r-\sigma^{2}\right)+\sqrt{\left(2 r-\sigma^{2}\right)^{2}+8 \lambda \sigma^{2}}}{2 \sigma^{2}}}-\varphi_{1}^{-\frac{\left(2 r-\sigma^{2}\right)+\sqrt{\left(2 r-\sigma^{2}\right)^{2}+8 \lambda \sigma^{2}}}{2 \sigma^{2}}} \varphi_{2}^{-\frac{\left(2 r-\sigma^{2}\right)-\sqrt{\left(2 r-\sigma^{2}\right)^{2}+8 \lambda \sigma^{2}}}{2 \sigma^{2}}}} \\
& \text { if } \lambda>-\frac{\left(2 r-\sigma^{2}\right)^{2}}{8 \sigma^{2}}
\end{aligned}
$$




$$
\begin{aligned}
& E^{\varphi}[\exp (-\lambda T)]=\left(\frac{\varphi}{\varphi_{1} \varphi_{2}}\right)^{\frac{\sigma^{2}-2 r}{2 \sigma^{2}}} \frac{\left(\ln \varphi_{2}-\ln \varphi\right) \varphi_{2}^{\frac{\sigma^{2}-2 r}{2 \sigma^{2}}}-\left(\ln \varphi-\ln \varphi_{1}\right) \varphi_{1}^{\frac{\sigma^{2}-2 r}{2 \sigma^{2}}}}{\ln \varphi_{2}-\ln \varphi_{1}} \text {, if } \lambda=\frac{\sigma^{2}-2 r}{2 \sigma^{2}} \\
& E^{\varphi}[\exp (-\lambda T)]=\left(\frac{\varphi}{\varphi_{1} \varphi_{2}}\right)^{\frac{\sigma^{2}-2 r}{2 \sigma^{2}}} \frac{\varphi_{2}^{\frac{\sigma^{2}-2 r}{2 \sigma^{2}}} \sin \left[\sqrt{-\left(2 r-\sigma^{2}\right)^{2}-8 \lambda \sigma^{2}} \ln \left(\frac{\varphi_{2}}{\varphi}\right)\right]}{\sin \left[\sqrt{-\left(2 r-\sigma^{2}\right)^{2}-8 \lambda \sigma^{2}} \ln \left(\frac{\varphi_{2}}{\varphi_{1}}\right)\right]}+ \\
& +\frac{\varphi_{1}^{\frac{\sigma^{2}-2 r}{\sigma^{2}}} \sin \left[\sqrt{-\left(2 r-\sigma^{2}\right)^{2}-8 \lambda \sigma^{2}} \ln \left(\frac{\varphi}{\varphi_{1}}\right)\right]}{\sin \left[\sqrt{-\left(2 r-\sigma^{2}\right)^{2}-8 \lambda \sigma^{2}} \ln \left(\frac{\varphi_{2}}{\varphi_{1}}\right)\right]} \\
& \text { if } \lambda<-\frac{\left(2 r-\sigma^{2}\right)^{2}}{8 \sigma^{2}}
\end{aligned}
$$

Proof. Reminder: Assume that $\lambda>-\frac{\lambda_{1}}{2}$, where $\lambda_{1}$ is the first Dirichlet eigenvalue of $D \subset S^{1}$. If $u(x)=E[\exp (-\lambda T)]$ then $u(x)$ satisfies

$L u(\varphi)=\lambda u(\varphi)$ with boundary conditions $\left.u\right|_{\partial D}=1$, (see [13])

The first dirichlet eigenvalue of $D \subset S^{1}$ is $\lambda=\frac{\pi^{2}}{a^{2}\left(\varphi_{2}-\varphi_{1}\right)^{2}}$. Hence if $\lambda>-\frac{\pi^{2}}{a^{2}\left(\varphi_{2}-\varphi_{1}\right)^{2}}$, then $E^{\varphi}[\exp (-\lambda T)]$ satisfies the differential equation

$$
r \varphi \frac{\partial u}{\partial \varphi}+\frac{\sigma^{2} \varphi^{2}}{2} \frac{\partial^{2} u}{\partial \varphi^{2}}=\lambda u(\varphi)
$$

with boundary condition

$$
u\left(\varphi_{1}\right)=u\left(\varphi_{2}\right)=1
$$

This is a Cauchy-Euler equation. The solution is

$E^{\varphi}[\exp (-\lambda T)]$

$$
\begin{aligned}
& =\frac{\left(\varphi_{2}^{-\frac{\left(2 r-\sigma^{2}\right)+\sqrt{\left(2 r-\sigma^{2}\right)^{2}+8 \lambda \sigma^{2}}}{2 \sigma^{2}}}-\varphi_{1}^{-\frac{\left(2 r-\sigma^{2}\right)+\sqrt{\left(2 r-\sigma^{2}\right)^{2}+8 \lambda \sigma^{2}}}{2 \sigma^{2}}}\right) \varphi^{-\frac{\left(2 r-\sigma^{2}\right)-\sqrt{\left(2 r-\sigma^{2}\right)^{2}+8 \lambda \sigma^{2}}}{2 \sigma^{2}}}}{\varphi_{1}^{-\frac{\left(2 r-\sigma^{2}\right)-\sqrt{\left(2 r-\sigma^{2}\right)^{2}+8 \lambda \sigma^{2}}}{2 \sigma^{2}}} \varphi_{2}^{-\frac{\left(2 r-\sigma^{2}\right)+\sqrt{\left(2 r-\sigma^{2}\right)^{2}+8 \lambda \sigma^{2}}}{2 \sigma^{2}}}-\varphi_{1}^{-\frac{\left(2 r-\sigma^{2}\right)+\sqrt{\left(2 r-\sigma^{2}\right)^{2}+8 \lambda \sigma^{2}}}{2 \sigma^{2}}} \varphi_{2}^{-\frac{\left(2 r-\sigma^{2}\right)-\sqrt{\left(2 r-\sigma^{2}\right)^{2}+8 \lambda \sigma^{2}}}{2 \sigma^{2}}}} \\
& +\frac{\left(\varphi_{1}^{-\frac{\left(2 r-\sigma^{2}\right)-\sqrt{\left(2 r-\sigma^{2}\right)^{2}+8 \lambda \sigma^{2}}}{2 \sigma^{2}}}-\varphi_{2}^{\left.-\frac{\left(2 r-\sigma^{2}\right)-\sqrt{\left(2 r-\sigma^{2}\right)^{2}+8 \lambda \sigma^{2}}}{2 \sigma^{2}}\right)} \varphi^{-\frac{\left(2 r-\sigma^{2}\right)+\sqrt{\left(2 r-\sigma^{2}\right)^{2}+8 \lambda \sigma^{2}}}{2 \sigma^{2}}}\right.}{\varphi_{1}^{-\frac{\left(2 r-\sigma^{2}\right)-\sqrt{\left(2 r-\sigma^{2}\right)^{2}+8 \lambda \sigma^{2}}}{2 \sigma^{2}}} \varphi_{2}^{-\frac{\left(2 r-\sigma^{2}\right)+\sqrt{\left(2 r-\sigma^{2}\right)^{2}+8 \lambda \sigma^{2}}}{2 \sigma^{2}}}-\varphi_{1}^{-\frac{\left(2 r-\sigma^{2}\right)+\sqrt{\left(2 r-\sigma^{2}\right)^{2}+8 \lambda \sigma^{2}}}{2 \sigma^{2}}} \varphi_{2}^{-\frac{\left(2 r-\sigma^{2}\right)-\sqrt{\left(2 r-\sigma^{2}\right)^{2}+8 \lambda \sigma^{2}}}{2 \sigma^{2}}}} \\
& \text { if } \lambda>-\frac{\left(2 r-\sigma^{2}\right)^{2}}{8 \sigma^{2}}
\end{aligned}
$$$$
E^{\varphi}[\exp (-\lambda T)]=\left(\frac{\varphi}{\varphi_{1} \varphi_{2}}\right)^{\frac{\sigma^{2}-2 r}{2 \sigma^{2}}} \frac{\left(\ln \varphi_{2}-\ln \varphi\right) \varphi_{2}^{\frac{\sigma^{2}-2 r}{2 \sigma^{2}}}-\left(\ln \varphi-\ln \varphi_{1}\right) \varphi_{1}^{\frac{\sigma^{2}-2 r}{2 \sigma^{2}}}}{\ln \varphi_{2}-\ln \varphi_{1}}, \text { if } \lambda=\frac{\sigma^{2}-2 r}{2 \sigma^{2}}
$$ 


$$
\begin{aligned}
E^{\varphi}[\exp (-\lambda T)]= & \left(\frac{\varphi}{\varphi_{1} \varphi_{2}}\right)^{\frac{\sigma^{2}-2 r}{2 \sigma^{2}}} \frac{\varphi_{2}^{\frac{\sigma^{2}-2 r}{2 \sigma^{2}}} \sin \left[\sqrt{-\left(2 r-\sigma^{2}\right)^{2}-8 \lambda \sigma^{2}} \ln \left(\frac{\varphi_{2}}{\varphi}\right)\right]}{\sin \left[\sqrt{-\left(2 r-\sigma^{2}\right)^{2}-8 \lambda \sigma^{2}} \ln \left(\frac{\varphi_{2}}{\varphi_{1}}\right)\right]}+ \\
& +\frac{{\frac{\sigma^{2}-2 r}{2 \sigma^{2}}}^{2} \sin \left[\sqrt{-\left(2 r-\sigma^{2}\right)^{2}-8 \lambda \sigma^{2}} \ln \left(\frac{\varphi}{\varphi_{1}}\right)\right]}{\sin \left[\sqrt{-\left(2 r-\sigma^{2}\right)^{2}-8 \lambda \sigma^{2}} \ln \left(\frac{\varphi_{2}}{\varphi_{1}}\right)\right]} \\
\text { if } \lambda< & -\frac{\left(2 r-\sigma^{2}\right)^{2}}{8 \sigma^{2}}
\end{aligned}
$$

i.e. if $Y_{t}$ is the asset price and we have a double knock-out Barrier at levels $H_{1}=a \varphi_{1}$ and $H_{2}=a \varphi_{2}$, then if its price starts at the point $x \in\left(H_{1}, H_{2}\right)$

$E^{x}[\exp (-\lambda T)]=$

$$
\begin{aligned}
& \left(H_{2}^{-\frac{\left(2 r-\sigma^{2}\right)+\sqrt{\left(2 r-\sigma^{2}\right)^{2}+8 \lambda \sigma^{2}}}{2 \sigma^{2}}}-H_{1}^{-\frac{\left(2 r-\sigma^{2}\right)+\sqrt{\left(2 r-\sigma^{2}\right)^{2}+8 \lambda \sigma^{2}}}{2 \sigma^{2}}}\right) x^{-\frac{\left(2 r-\sigma^{2}\right)-\sqrt{\left(2 r-\sigma^{2}\right)^{2}+8 \lambda \sigma^{2}}}{2 \sigma^{2}}} \\
& H_{1}^{-\frac{\left(2 r-\sigma^{2}\right)-\sqrt{\left(2 r-\sigma^{2}\right)^{2}+8 \lambda \sigma^{2}}}{2 \sigma^{2}}} H_{2}^{-\frac{\left(2 r-\sigma^{2}\right)+\sqrt{\left(2 r-\sigma^{2}\right)^{2}+8 \lambda \sigma^{2}}}{2 \sigma^{2}}}-H_{1}^{-\frac{\left(2 r-\sigma^{2}\right)+\sqrt{\left(2 r-\sigma^{2}\right)^{2}+8 \lambda \sigma^{2}}}{2 \sigma^{2}}} H_{2}^{-\frac{\left(2 r-\sigma^{2}\right)-\sqrt{\left(2 r-\sigma^{2}\right)^{2}+8 \lambda \sigma}}{2 \sigma^{2}}}
\end{aligned}
$$

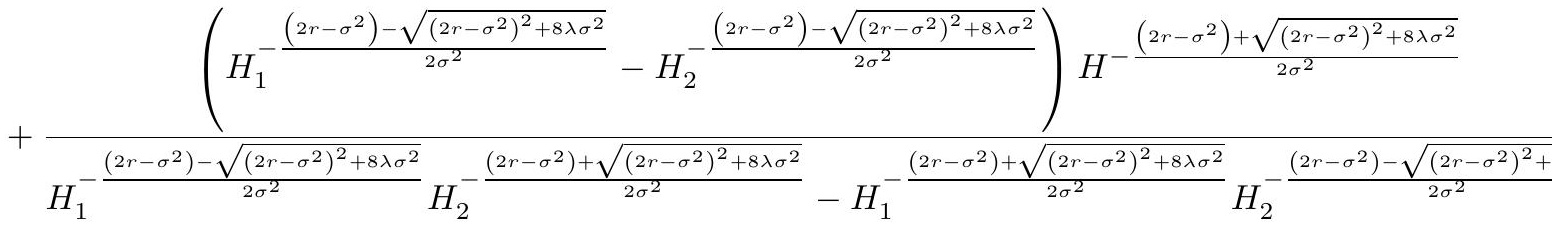

$$
\begin{aligned}
& \text { if } \lambda>-\frac{\left(2 r-\sigma^{2}\right)^{2}}{8 \sigma^{2}} \\
& E^{x}[\exp (-\lambda T)]=\left(\frac{x}{H_{1} H_{2}}\right)^{\frac{\sigma^{2}-2 r}{2 \sigma^{2}}} \frac{\left(\ln H_{2}-\ln x\right) H_{2}^{\frac{\sigma^{2}-2 r}{2 \sigma^{2}}}-\left(\ln x-\ln H_{1}\right) H_{1}^{\frac{\sigma^{2}-2 r}{2 \sigma^{2}}}}{\ln H_{2}-\ln H_{1}} \text { if } \lambda=\frac{\sigma^{2}-2 r}{2 \sigma^{2}} \\
& E^{x}[\exp (-\lambda T)]=\left(\frac{x}{H_{1} H_{2}}\right)^{\frac{\sigma^{2}-2 r}{2 \sigma^{2}}} \frac{H_{2}^{\frac{\sigma^{2}-2 r}{2 \sigma^{2}}} \sin \left[\sqrt{-\left(2 r-\sigma^{2}\right)^{2}-8 \lambda \sigma^{2}} \ln \left(\frac{H_{2}}{x}\right)\right]}{\sin \left[\sqrt{-\left(2 r-\sigma^{2}\right)^{2}-8 \lambda \sigma^{2}} \ln \left(\frac{H_{2}}{H_{1}}\right)\right]}+ \\
& +\frac{H_{1}^{\frac{\sigma^{2}-2 r}{2 \sigma^{2}}} \sin \left[\sqrt{-\left(2 r-\sigma^{2}\right)^{2}-8 \lambda \sigma^{2}} \ln \left(\frac{x}{H_{1}}\right)\right]}{\sin \left[\sqrt{-\left(2 r-\sigma^{2}\right)^{2}-8 \lambda \sigma^{2}} \ln \left(\frac{H_{2}}{H_{1}}\right)\right]} \\
& \text { if } \lambda<-\frac{\left(2 r-\sigma^{2}\right)^{2}}{8 \sigma^{2}}
\end{aligned}
$$

\section{Local Time estimation}

Definition 5.1. Let $\varphi_{0}, \varphi_{1} \in(0,2 \pi]$, such that $\varphi_{0}<\varphi_{1}$ both fixed. We consider the set $D$ in $S^{1}$ such that $D=\left(\varphi_{0}, \varphi_{1}\right)$. The Reflected Geometric Brownian Motion in $D$ is the diffusion $W_{t}$ whose generator is $L$ in $D$ with Neuman boundary condition at $\partial D$.

Roughly speaking $W_{t}$ behaves like $Z_{t}$ inside $D$ but when it reaches the boundary it is reflected back in $D$. 
Definition 5.2. Let a fixed open set $D \subset S^{1}$ with $C^{3}$-boundary $\partial D$. If $Y_{t}$ is the Reflected Geometric Brownian Motion in $D$, and $D_{\delta}$ the domain

$$
D_{\delta}=\{x \in D \mid d(x, \partial D)<\delta\}
$$

we define the boundary local time $L_{t}$ of $W_{t}$ as

$$
L_{t}=\lim _{\delta \rightarrow 0} \frac{1}{2 \delta} \int_{0}^{t} 1_{D_{\delta}}\left(W_{s}\right) d s
$$

It can be shown that the limit exists in $L_{2}$ sense.

\subsection{Boundary Local Time Until First Hitting}

Proposition 5.1. Let $\varphi_{1}, \varphi_{2} \in(0,2 \pi]$, such that $\varphi_{1}<\varphi_{2}$ both fixed. We consider the set $D$ in $S^{1}$ such that $D=\left(\varphi_{1}, \varphi_{2}\right)$. Let $W_{t}$ be the The Reflected Geometric Brownian Motion on D starting at the point $\varphi \in D$. If

$$
T=\inf \left\{t \geq 0 \mid Z_{t}=\varphi_{1}\right\}
$$

and $L_{t}$ is the boundary local time of $W_{t}$, then

$$
E^{\varphi}\left[\exp \left(\lambda L_{t}\right)\right]=\frac{\left(\sigma^{2}-2 r\right) \varphi_{2}^{-\frac{2 r}{\sigma^{2}}}-\lambda \sigma^{2}\left(\varphi_{2}^{\frac{\sigma^{2}-2 r}{\sigma^{2}}}-\varphi^{\frac{\sigma^{2}-2 r}{\sigma^{2}}}\right)}{\left(\sigma^{2}-2 r\right) \varphi_{2}^{-\frac{2 r}{\sigma^{2}}}-\lambda \sigma^{2}\left(\varphi_{2}^{\frac{\sigma^{2}-2 r}{\sigma^{2}}}-\varphi_{1}^{\frac{\sigma^{2}-2 r}{\sigma^{2}}}\right)}, \quad \text { if } \quad \lambda<\frac{\left(\sigma^{2}-2 r\right) \varphi_{2}^{-\frac{2 r}{\sigma^{2}}}}{\sigma^{2}\left(\varphi_{2}^{\frac{\sigma^{2}-2 r}{\sigma^{2}}}-\varphi_{1}^{\frac{\sigma^{2}-2 r}{\sigma^{2}}}\right)}
$$

and

$$
E^{\varphi}\left[\exp \left(\lambda L_{t}\right)\right]=+\infty, \quad \text { if } \quad \lambda \geq \frac{\left(\sigma^{2}-2 r\right) \varphi_{2}^{-\frac{2 r}{\sigma^{2}}}}{\sigma^{2}\left(\varphi_{2}^{\frac{\sigma^{2}-2 r}{\sigma^{2}}}-\varphi_{1}^{\frac{\sigma^{2}-2 r}{\sigma^{2}}}\right)}
$$

Proof. It is known that the function $Z(\varphi)=E^{\varphi}\left[\exp \left(\lambda L_{t}\right)\right]$ satisfies the differential equation

$$
L z=0
$$

with boundary conditions

$$
z\left(\varphi_{1}\right)=1
$$

and

$$
-\frac{d z}{d \varphi}\left(\varphi_{2}\right)+\lambda z\left(\varphi_{2}\right)=0
$$

as long as the function $z$ is positive (see [15])

Thus

$$
z(\varphi)=\frac{\left(\sigma^{2}-2 r\right) \varphi_{2}^{-\frac{2 r}{\sigma^{2}}}-\lambda \sigma^{2}\left(\varphi_{2}^{\frac{\sigma^{2}-2 r}{\sigma^{2}}}-\varphi^{\frac{\sigma^{2}-2 r}{\sigma^{2}}}\right)}{\left(\sigma^{2}-2 r\right) \varphi_{2}^{-\frac{2 r}{\sigma^{2}}}-\lambda \sigma^{2}\left(\varphi_{2}^{\frac{\sigma^{2}-2 r}{\sigma^{2}}}-\varphi_{1}^{\frac{\sigma^{2}-2 r}{\sigma^{2}}}\right)}
$$

However $z(\varphi)>0$ for every $\varphi \in\left(\varphi_{1}, \varphi_{2}\right)$ if and only if $\lambda<\frac{\left(\sigma^{2}-2 r\right) \varphi_{2}^{-\frac{2 r}{\sigma^{2}}}}{\sigma^{2}\left(\varphi_{2}^{\frac{\sigma^{2}-2 r}{\sigma^{2}}}-\varphi_{1}^{\frac{\sigma^{2}-2 r}{\sigma^{2}}}\right)}$. Therefore

$$
E^{\varphi}\left[\exp \left(\lambda L_{t}\right)\right]=\frac{\left(\sigma^{2}-2 r\right) \varphi_{2}^{-\frac{2 r}{\sigma^{2}}}-\lambda \sigma^{2}\left(\varphi_{2}^{\frac{\sigma^{2}-2 r}{\sigma^{2}}}-\varphi^{\frac{\sigma^{2}-2 r}{\sigma^{2}}}\right)}{\left(\sigma^{2}-2 r\right) \varphi_{2}^{-\frac{2 r}{\sigma^{2}}}-\lambda \sigma^{2}\left(\varphi_{2}^{\frac{\sigma^{2}-2 r}{\sigma^{2}}}-\varphi_{1}^{\frac{\sigma^{2}-2 r}{\sigma^{2}}}\right)}, \quad \text { if } \quad \lambda<\frac{\left(\sigma^{2}-2 r\right) \varphi_{2}^{-\frac{2 r}{\sigma^{2}}}}{\sigma^{2}\left(\varphi_{2}^{\frac{\sigma^{2}-2 r}{\sigma^{2}}}-\varphi_{1}^{\frac{\sigma^{2}-2 r}{\sigma^{2}}}\right)}
$$


and

$$
E^{\varphi}\left[\exp \left(\lambda L_{t}\right)\right]=+\infty, \quad \text { if } \quad \lambda \geq \frac{\left(\sigma^{2}-2 r\right) \varphi_{2}^{-\frac{2 r}{\sigma^{2}}}}{\sigma^{2}\left(\varphi_{2}^{\frac{\sigma^{2}-2 r}{\sigma^{2}}}-\varphi_{1}^{\frac{\sigma^{2}-2 r}{\sigma^{2}}}\right)}
$$

As a consequence of the proof, if we set $\tilde{\lambda}=a \lambda, Y_{t}$ the asset price and we consider the double knock-out barrier at levels $H_{1}=a \varphi_{1}$ and $H_{2}=a \varphi_{2}$ then if its price starts at the point $x \in\left(H_{1}, H_{2}\right)$, then the expectation is given as

$$
E^{x}\left[\exp \left(\tilde{\lambda} L_{t}\right)\right]=\frac{\left(\sigma^{2}-2 r\right) H_{2}^{-\frac{2 r}{\sigma^{2}}}-\tilde{\lambda} \sigma^{2}\left(H_{2}^{\frac{\sigma^{2}-2 r}{\sigma^{2}}}-x^{\frac{\sigma^{2}-2 r}{\sigma^{2}}}\right)}{\left(\sigma^{2}-2 r\right) H_{2}^{-\frac{2 r}{\sigma^{2}}}-\tilde{\lambda} \sigma^{2}\left(H_{2}^{\frac{\sigma^{2}-2 r}{\sigma^{2}}}-H_{1}^{\frac{\sigma^{2}-2 r}{\sigma^{2}}}\right)}, \quad \text { if } \quad \tilde{\lambda}<\frac{\left(\sigma^{2}-2 r\right) H_{2}^{-\frac{2 r}{\sigma^{2}}}}{\sigma^{2}\left(H_{2}^{\frac{\sigma^{2}-2 r}{\sigma^{2}}}-H_{1}^{\frac{\sigma^{2}-2 r}{\sigma^{2}}}\right)}
$$

and

$$
E^{x}\left[\exp \left(\tilde{\lambda} L_{t}\right)\right]=+\infty, \quad \text { if } \quad \tilde{\lambda} \geq \frac{\left(\sigma^{2}-2 r\right) H_{2}^{-\frac{2 r}{\sigma^{2}}}}{\sigma^{2}\left(H_{2}^{\frac{\sigma^{2}-2 r}{\sigma^{2}}}-H_{1}^{\frac{\sigma^{2}-2 r}{\sigma^{2}}}\right)}
$$

where $L_{t}$ is the boundary local time of the option price .

\section{Conclusion}

Our novel methodology is not restricted to underlying processes which are geometric Brownian motions on $S^{1}$. Any other form of underlying process can be used, provided that there exists a transformation between the process and standard Brownian motion on $S^{1}$. Moreover, we can easily extend all the above results on spheres of higher dimensions. Our approach can be applied to the valuation of other exotic derivatives as well as to other mathematical problems. For example, Brownian motions on $S^{2}$ can be utilized for other types of derivatives' pricing in financial literature, epidemiological models and enviromental pollution models among other. Also for $n=3, S^{3}$ some results appear in relativity theory ([16]). We contributed in a plethora of ways. In particular, we presented new ways of estimating the expectation of the time the options seize to exist, their hitting probabilities, the exit times and their expectations, boundary local times until the first hitting and other probabilistic quantities related to the boundary local times. We deliver closed-form solutions which maybe of immense importance to traders, investors, speculators and more broadly to financial institutions.

\section{References}

[1] Luo, L. 2001. Various types of double barrier options. Journal of Computational Finance 4, 125-138

[2] Merton R, C. 1973. Theory of rational option pricing Bell J. Econ. Management Science 4, 141-83

[3] Rubinstein M, Reiner, E. 1991. Breaking down the barriers. Risk 4, 28-35

[4] Rich, D R. 1994. The mathematical foundations of barrier option-pricing theory. Adv. Futures Opt. Res. 7, 267-311

[5] Kunitomo N, Ikeda, M. 1992. Pricing options with curved boundaries. Mathematical Finance 2, $275-98$ 
[6] Hui, C. H. 1996. One-touch double barrier binary option values. Appled Financial Economics 6, 343-6

[7] Hui, C H. 1997. Time-dependent barrier option values. Journal of Futures Markets 17, 667-88

[8] Roberts, G. O. Shortland, C. F. 1997. Pricing Barrier Options with Time-Dependent Coefficients. Mathematical Finance 7,: 83-93

[9] Dell'Era Mario, M. D. 2008. Pricing of Double Barrier Options by Spectral Theory. MPRA Paper 17502, University Library of Munich, Germany.

[10] Do Carmo, M.P. 1976. Differential Geometry of Curves and Surfaces Prentice-Hall, Inc. Upper Saddle River, New Jersey

[11] Dodziuk J., Maximum Principle for Parabolic Inequalities and the Heat Flow on Open Manifolds, Indiana Oniv. Math. J. 32 no.5 115-142 (1983).

[12] Harrison, J. M., Pliska, S. R. 1981. Martingales and Stochastic Integrals in the Theory of Continuous Trading.Stoch. Process Appl. 11, 215-260.

[13] Oksendal, B., 1995. Stochastic Differential Equations. Springer-Verlag.

[14] Dynkin, E.B.1965. Markov processes. Vol. 2 Springer, Berlin (1965)

[15] Papanicolaou V.G., 1990. The probabilistic solutions of the third boundary value problem for second order elliptic equations. Probability Theory and related fields 87, 27-77

[16] Kouloumpou D., Papanicolaou, V. G., 2013. Certain Calculation Regarding the Brownian Motion on the Sphere. Journal of Concrete and Applicable Mathematics 11, 26-41. 